\title{
TITLE:
}

\section{Control of cell attachment through polyDNA hybridization.}

\section{AUTHOR(S):}

Teramura, Yuji; Chen, Hao; Kawamoto, Takuo; Iwata, Hiroo

\section{CITATION:}

Teramura, Yuji ... [et al]. Control of cell attachment through polyDNA hybridization.. Biomaterials 2010, 31(8): 2229-2235

\section{ISSUE DATE:}

2010-03

URL:

http://hdl.handle.net/2433/95080

\section{RIGHT:}

(c) 2009 Elsevier Ltd All rights reserved.; This is not the published version. Please cite only the published version.; この論文は出版社版でありませ ん。引用の際には出版社版をご確認ご利用ください。 


\title{
Control of cell attachment through polyDNA hybridization
}

\author{
${ }^{a}$ Radioisotope Research Center, Kyoto University, Yoshida-Konoe-Cho, Sakyo-ku, \\ Kyoto, 606-8501, Japan, ${ }^{\mathrm{b}}$ Department of Reparative Materials, Institute for Frontier \\ Medical Sciences, Kyoto University, 53 Kawara-Cho, Shogoin, Sakyo-ku, Kyoto, \\ 606-8507, Japan. \\ * Address correspondence and reprint requests to Hiroo Iwata, Ph.D. \\ E-mail: iwata@frontier.kyoto-u.ac.jp, PHONE/FAX: +81-75-751-4119
}




\begin{abstract}
Cell-cell interactions play vital roles in embryo development and in homeostasis maintenance. Such interactions must be stringently controlled for cell-based tissue engineering and regenerative medicine therapies, and methods for studying and controlling cell-cell interactions are being developed using both biomedical and engineering approaches. In this study, we prepared amphiphilic PEG-lipid polymers that were attached to polyDNA with specific sequences. Incubation of cells with the polyDNA-PEG-lipid conjugate transferred some of the polyDNA to the cells' surfaces. Similarly, polyDNA-PEG-lipid conjugate using polyDNA with a complementary sequence was introduced to the surfaces of other cells or to a substrate surface. Cell-cell or cell-substrate attachments were subsequently mediated via hybridization between the two complementary polyDNAs and monitored using fluorescence microscopy.
\end{abstract}

Keywords: Cell-cell attachment; poly(ethylene glycol)-lipid (PEG-lipid); DNA hybridization; Surface modification. 


\section{Introduction}

In the past decade, therapeutic devices containing living cells or tissues have been studied extensively for tissue engineering and regenerative medicine applications. Stem cells, including embryonic stem (ES) cells, somatic stem cells, and induced pluripotent stem (iPS) cells, have been identified and studied [1-3] that show promise for treatment of diseases such as type I diabetes, Parkinson's, Alzheimer's, ALS, and Huntington's disease [4-11]. Experimental manipulation of cell-cell interactions is a valuable method for inducing differentiation of stem cells for use in cell-based therapies. In addition, the differentiated cells can be manipulated further for use in regenerating tissues or organs. Cell-cell interactions must be tightly controlled for generating cell-type-specific tissues or organs. Cell-cell interactions are also used to develop pluripotent stem cells themselves. It was reported recently that somatic cells could be transformed into pluripotent stem cells by fusion with ES cells [12]. In this method, somatic cells and ES cell attachments formed first, and attachment was followed by induced cell fusion. Cell-cell interactions are also very important in embryo development and in the maintenance of homeostasis. Methods for studying and controlling cell-cell interactions are currently being developed using both biomedical and engineering approaches. Our group has studied the surface modification of living cells using amphiphilic polymers 
such as PEG-conjugated phospholipid (PEG-lipid) derivatives [13-19]. Specifically, our

previous efforts were directed towards modification of cell surfaces and islets of

Langerhans (islets) by introducing functional groups and polymers for improving graft

survival after transplantation. Recently, immobilization of cells to the surface of islets

using PEG-lipid and a biotin/streptavidin reaction resulted in encapsulation of the whole

islet surface with layers of cells [19]. It seemed possible to use this method to induce

cells to attach to a substrate. Although the biotin/streptavidin reaction is well

characterized and is used frequently in biological studies, it has some disadvantages.

Specifically, streptavidin is derived from bacteria and is a potent antigen in humans;

further, the biotin/streptavidin association is so strong that it is difficult to be

dissociated.

In the present study, we employed DNA hybridization rather than the biotin/streptavidin reaction as a novel method for inducing cell-cell attachment and cell immobilization on a substrate. We used PEG-lipid, which is an amphiphilic polymer, as a carrier for polyDNA with a specific sequence. Cells treated with the polyDNA-PEG-lipid conjugate incorporated the lipid (and thus the polyDNA) onto the cell surface. PolyDNA with the complementary sequence was similarly transferred onto the surface 
1

2

3

4

5

6

7

8

9

10

11

12

13

14

15

16

17

18

19

20

21

22

23

24

25

26

27

28

29

30

31

32

33

34

35

36

37

38

39

40

41

42

43

44

45

46

47

48

49

50

51

52

53

54

55

56

57

58

59

60

61

62

63

64

65

or other cells or onto a substrate. Cell-cell or cell-substrate attachments were

subsequently induced via hybridization between the two complementary polyDNAs. 


\section{Materials and Methods}

\subsection{Materials}

$\alpha-N$-Hydroxysuccinimidyl- $\omega$-maleimidyl poly(ethylene glycol) (NHS-PEG-Mal, MW:

5000) was from Nektar Therapeutics (San Carlos, CA, USA).

1,2-dipalmitoyl-sn-glycerol-3-phosphatidylethanolamine (DPPE) was from NOF

Corporation (Tokyo, Japan). Dichloromethane, triethylamine, and diethyl ether was

from Nacalai Tesque (Kyoto, Japan). Hanks' balanced salt solution (HBSS), minimum

essential medium (MEM), and RPMI-1640 medium were from Invitrogen Co.

(Carlsbad, CA, USA). Fetal bovine serum (FBS) was from Equitech-Bio, Inc. (TX,

USA), and phosphate-buffered saline (PBS) was from Nissui Pharmaceutical Co. Ltd.

(Tokyo, Japan). PKH67 Green Fluorescent Cell Linker Kit (PKH green) and PKH26

Red Fluorescent Cell Linker Kit (PKH red) were from Sigma-Aldrich Chemical Co. (St.

Louis, MO, USA). $n$-Hexadecyl mercaptan was from Tokyo Chemical Industry Co., Ltd

(Tokyo, Japan). Glass plates (22 mm x $26 \mathrm{~mm}$; thickness: 0.12-0.17 mm) were from

Matsunami Glass Ind., Ltd (Osaka, Japan). Dithiothreitol (DTT) was from Wako Pure

Chemical Industries, Ltd (Osaka, Japan).

\subsection{Synthesis of DNA-conjugated PEG-phospholipid (polyDNA-PEG-lipid)}


Mal-PEG-lipid was synthesized by combining NHS-PEG-Mal (180 mg), triethylamine

$(50 \mu \mathrm{L})$, and DPPE (20 mg) with dichloromethane and stirring for $36 \mathrm{~h}$ at room temperature (RT) [14]. After precipitation with diethyl ether, Mal-PEG-lipid was obtained as a white powder (190 mg, 80\% yield). ${ }^{1} \mathrm{H}-\mathrm{NMR}$ analysis $\left(\mathrm{CDCl}_{3}, 400 \mathrm{MHz}\right.$, $\delta$ ppm): 0.88 (t, 6H, - $\left.\mathrm{CH}_{3}\right), 1.25$ (br, 56H, - $\left.\mathrm{CH}_{2^{-}}\right) 3.64$ (br, 480H, PEG), 6.71 (s, 2H, $-\mathrm{HC}=\mathrm{CH}-$, maleimide).

The DNA sequences used in this study are listed in Table 1. DNA was synthesized by Sigma-Aldrich Chemical Co. DNA-SH was prepared by reduction of the disulfide bond with DTT according to the manufacturer's instructions. A PBS solution of DNA-SH $(1.0 \mathrm{mg})$ was mixed with Mal-PEG-lipid $(5.0 \mathrm{mg})$ in PBS for $24 \mathrm{~h}$ at RT to prepare polyDNA-PEG-lipid. PolyDNA-PEG-lipid (500 $\mu \mathrm{g} / \mathrm{mL}$ in PBS) was used for surface modification of cells without purification.

\subsection{Cell cultures}

Two cell lines, CCRF-CEM cells (a human T cell lymphoblast-like cell line) and HEK293 cells (a human embryonic kidney cell line) were obtained from the Health Science Research Resources Bank (Osaka, Japan). Suspension culture of CCRF-CEM cells was performed in RPMI-1640 medium supplemented with 10\% FBS, $100 \mathrm{U} / \mathrm{mL}$ 
penicillin, and $0.1 \mathrm{mg} / \mathrm{mL}$ streptomycin (Invitrogen) at $37{ }^{\circ} \mathrm{C}$ under $5 \% \mathrm{CO}_{2}$. HEK293

cells that stably expressed enhanced green fluorescence protein (EGFP) (GFP-HEK)

were the kind gift of Dr. K. Kato (Institute for Frontier Medical Sciences, Kyoto

University). The GFP-HEK cells were maintained in MEM supplemented with $10 \%$

FBS, $100 \mathrm{U} / \mathrm{mL}$ penicillin, and $0.1 \mathrm{mg} / \mathrm{mL}$ streptomycin.

\subsection{Surface modification of cells with polyDNA-PEG-lipid and co-incubation of differentially modified cells}

For visualization under a fluorescence microscope, CCRF-CEM cells were labeled with

PKH red or PKH green according to the manufacturer's instructions. To exchange the culture medium, CCRF-CEM or GFP-HEK cells $\left(4 \times 10^{6}\right.$ cells $)$ were washed twice with HBSS and collected by centrifugation $\left(180 \times g, 5 \mathrm{~min}, 25^{\circ} \mathrm{C}\right)$. After the addition of polyDNA-PEG-lipid solution $(50 \mu \mathrm{L}, 500 \mu \mathrm{g} / \mathrm{mL}$ in PBS) to the cell suspension, cells were incubated for $30 \mathrm{~min}$ at RT with gentle agitation. The cells were then suspended in $10 \mathrm{~mL}$ HBSS, collected by centrifugation $\left(180 \times g, 5 \mathrm{~min}, 25^{\circ} \mathrm{C}\right)$, washed with another $10 \mathrm{~mL}$ HBSS, and re-centrifuged to obtain polyDNA-PEG-lipid-modified cells.

After cells were treated with polyA-PEG-lipid or polyT-PEG-lipid, the polyDNA-PEG-lipid-modified cells were mixed together in culture medium with the 
following ratios of polyA-cells:polyT-cells: 10:1, 4:1, 2:1, and 1:1. The cells were incubated with rotation at $100 \mathrm{rpm}$ for $1 \mathrm{~h}$ at $\mathrm{RT}$, followed by incubation at $37^{\circ} \mathrm{C}$ under $5 \% \mathrm{CO}_{2}$. The cells were observed over time using a confocal laser scanning microscope (FLUOVIEW FV500, Olympus, Tokyo, Japan) and a phase-contrast microscope (IX7, Olympus Optical Co. Ltd., Tokyo, Japan).

\subsection{Immobilization of polyDNA-PEG-lipid modified cells to patterned substrates}

SeqA-conjugated PEG-lipid and SeqB-conjugated PEG-lipid were used for cell surface modification. For testing immobilization of the modified cells, substrate surfaces were modified using SeqA' and SeqB', the sequences complementary to SeqA and SeqB. Glass plates were cleaned with a piranha solution (7:3 mixture of concentrated sulfuric acid and 30\% hydrogen peroxide solution), washed 3x with Milli-Q water, and stored in a 2-propanol solution. For experiments, glass plates were mounted on a rotation stage in a metal vapor deposition apparatus (V-KS200, Osaka Vacuum Instruments, Osaka, Japan). A 1.0-nm chromium layer was deposited on the glass, followed by deposition of a 19-nm gold layer. The resulting glass plates coated with a thin layer of gold were immersed in an ethanol solution of $n$-hexadecyl mercaptan $(1 \mathrm{mM})$ to produce a surface with SAM-carrying methyl groups $\left(\mathrm{CH}_{3}-\mathrm{SAM}\right)$. The $\mathrm{CH}_{3}$-SAM surface was irradiated 
with an ultraviolet (UV) light at $180 \mathrm{~mW} / \mathrm{cm}^{2}$ using an Optical ModuleX (SX-UI

501HQ, Ushio, Inc., Tokyo) equipped with a super-high-pressure mercury lamp (Ushio,

Inc.) through a photomask with an array of transparent 1- or 2-mm circular dots in

ambient air for $4 \mathrm{~h}$. The plates were washed with ethanol to remove photodegradation

products. A PBS solution of DNA-SH (600 $\mu \mathrm{g} / \mathrm{mL}$, SeqA' and SeqB'), was applied to the UV-irradiated spots by manual pipetting and allowed to incubate for $2 \mathrm{~h}$ at RT. The substrate-coated glass plate was washed with HBSS before use.

In the first series of experiments, SeqA-PEG-lipid modified CCRF-CEM cells (SeqA-PEG-cells) and SeqB-PEG-lipid modified CCRF-CEM cells (SeqB-PEG-cells) were mixed at the following ratios: $4: 1,2: 1,1: 1,2: 1$, and $4: 1$. The cell suspensions were applied to UV-irradiated spots that had been incubated with a 1:1 mixture of SeqA' and SeqB' (see above); cells were incubated on the immobilized-DNA surface for $10 \mathrm{~min}$ at RT. In a second series of experiments, the UV-irradiated spots were incubated with SeqA':SeqB' at the following molar ratios: 4:1, 2:1, 1:1, 2:1, and 4:1. A 1:1 mixture of SeqA-PEG-cells and SeqB-PEG-cells was then applied to the UV-irradiated spots containing immobilized DNA. After washing with HBSS, cells attached to the substrate were observed using an upright fluorescence microscope (BX51, Olympus, Tokyo, 
Japan) and a stereomicroscope (MZF LIII, Leica, Solms, Germany). The number of attached cells was analyzed using ImageJ software (NIH, Bethesda, MD, USA).

An inhibition assay was also performed using a solution of SeqA' $(200 \mu \mathrm{g} / \mathrm{mL})$ that was added to the mixture of SeqA-PEG-cells and SeqB-PEG-cells. After incubation for 30 min, the mixture was applied to the SeqA' and SeqB'-immobilized substrate and incubated for 10 min at RT. After washing with HBSS, the cells attached to the substrate were observed using an upright fluorescence microscope.

Substrates for cell attachment were also prepared using a contact printing technique.

Poly(dimethylsiloxane) (PDMS) stamps were prepared as follows: A ledge pattern was fabricated on a PDMS surface using a laser beam machine (VLS2.30, Universal Laser Systems, Inc., Scottsdale, AZ, USA): The pattern consisted of unidirectional ledges (1 $\mathrm{mm} \times 1 \mathrm{~mm} \times 10 \mathrm{~mm}$ ) with $1-\mathrm{mm}$ intervals between ledges. The ledge surfaces on the stamps were coated with a solution of SeqA' or SeqB' DNA-SH $(600 \mu \mathrm{g} / \mathrm{mL})$ and applied to the gold-layered glass plates. A second stamp coated with a solution of SeqA' or SeqB' DNA-SH was applied to the surface perpendicular to the previous ledge design. The glass plate sat at RT for $2 \mathrm{~h}$ to dry. The glass plate was then immersed in an ethanol solution of $n$-hexadecyl mercaptan for blocking with $\mathrm{CH}_{3}$-SAM and washed with ethanol and Milli-Q water. A 1:1 mixture of SeqA-PEG-cells and SeqB-PEG-cells 
1

2

were applied onto the patterned substrate and incubated for 10 min at RT with gentle agitation. After washing with HBSS, cells attached to the glass plate were observed using an upright fluorescence microscope. 


\section{Results}

\subsection{Intercellular attachment through hybridization of complementary}

\section{polyDNA-PEG-lipid conjugates}

Scheme 1 shows how cells carrying complementary polyDNA-PEG-lipid conjugates were tested for intracellular attachment. polyDNA-PEG-lipids were synthesized using a thiol/maleimide reaction between Mal-PEG-lipid and DNA-SH in which the SH group was introduced at the 5'-end of the DNA sequence. The DNA sequences used in this study are listed in Table 1. PolyDNA-PEG-lipids carrying complementary sequences were prepared: polyA20 and polyT20, SeqA and SeqA', SeqB and SeqB'. Our previous studies demonstrated that amphiphilic PEG-lipids are spontaneously incorporated into the cell membrane's lipid bilayer through hydrophobic interactions and that this incorporation has no cytotoxic effects $[13-16,18,19]$. We further showed that polyDNA could be introduced onto the cell surface using a PEG-lipid (Scheme 1(b)). The strategy in the present study was to mediate cell-cell interactions by hybridization between complementary DNA sequences that were incorporated into the cells' outer membranes (Scheme 1(c)). Incorporation of polyA20-PEG-lipid into the cell membrane and its ability to hybridize with FITC-labeled polyT20 was examined first. A solution of polyA20-PEG-lipid was 
added to CCRF-CEM cells; after incubation, the cells were washed to remove

unincorporated lipid, FITC-labeled polyT20 was added, and cells were observed using a confocal laser scanning microscope. As shown in Fig. 1(a), the FITC fluorescence was observed at the periphery of all cells, indicating that polyA20-PEG-lipids were incorporated into the outer cell membrane and that FITC-labeled polyT20 hybridized with the incorporated polyA20 DNA. When FITC-labeled polyA20 was added to polyA20-PEG-lipid modified cells, no fluorescence was observed on the cells. These results indicated that FITC-labeled polyT20 hybridized specifically with polyA20-PEG-lipids on the cell surface.

Intercellular attachments could also be mediated by hybridization between polyA20 and polyT20, as shown in Fig. 1(c). CCRF-CEM cells labeled with PKH red were treated with polyA20-PEG-lipids (polyA20-PEG cells) and CCRF-CEM cells labeled with PKH green were treated with polyT20-PEG-lipids (polyT20-PEG-cells). Red polyA20-PEG-cells and green polyT20-PEG-cells were mixed at ratio of 1:1 and observed over time by a confocal laser scanning microscope (Fig. 1(c)). At 15 min after mixing, polyA20-PEG cells (red) and polyT20-PEG-cells (green) were attached to each other, with several cells attached in a linear fashion. At 60 min, even more cells had attached to each other. At $3 \mathrm{~h}$, the linear cell aggregates had gathered to form clumps of 
cells. At $6 \mathrm{~h}$, the cellular clumps were still present in the culture medium. As a control

experiment, PKH red- and PKH green-labeled cells with no polyDNA-PEG-lipid

treatment were mixed. These cells showed no attachment to each other (Fig. 1(e)). In

addition, there was no self attachment between polyT20-PEG-cells. These results

clearly showed that the attachment of different cells could be induced by hybridization

between polyA20 DNA and polyT20 DNA on the cell surfaces. The ratio of the number of attachments between polyA20-PEG-cells and polyT20-PEG-cells to the total number of attachments for all cells was approximately 1 at 15 and 60 min of incubation, indicating the alternating attachment of polyA20-PEG-cells and polyT20-PEG-cells. At $3 \mathrm{~h}$, the ratio had decreased to approximately 0.6 , indicating that larger aggregates of cells had formed. Cell-cell attachments could also be induced between polyA20-PEG-lipid modified CCRF-CEM cells (red) and polyT20-PEG-lipid modified GFP-HEK cells (green), as seen in Fig. 1(d). In contrast, no cell-cell attachments were observed between CCRF-CEM cells and GFP-HEK cells without polyDNA-PEG-lipid modification (Fig. 1(e)). Thus, this method can be used to promote attachments between different kinds of cells. 


\subsection{Attachment of polyDNA-PEG-cells to complementary DNA immobilized on a}

\section{solid substrate}

Glass plates with a thin layer of gold were modified with $\mathrm{CH}_{3}-\mathrm{SAM}$ and irradiated with

UV light through a photomask with an array of 1- or 2-mm transparent circular dots.

After washing the plates to remove photodegradation products, a solution containing

DNA-SH was spotted on the dots in order to immobilize DNA via the Au/thiol reaction

(Fig. 2(a)). PolyT20-PEG-cells labeled with PKH green were placed on the 2-mm spots where polyA20 molecules were immobilized and incubated for 10 min. After removal of unattached cells by washing with HBSS, the surface was observed using an upright fluorescence microscope. As shown in Fig. 2(b), polyT20-PEG-cells attached to the polyA20-immobilized spot. Fig 2(c) shows attachment of polyT20-PEG-cells onto a substrate with polyA20-SH and polyT20-SH spots. After polyT20-PEG-cells labeled with PKH green were applied and incubated for $10 \mathrm{~min}$, and unattached cells were washed off with HBSS, the substrate was observed using a stereomicroscope (Fig. 2(c)). PolyT20-PEG-cells selectively attached to the polyA20-immobilized spots, with practically no attachment of cells to the polyT20-immobilized spots (dotted lines). These results showed that cells attached to the substrate through hybridization of DNA on the cell surface and on the substrate. 
Next, a similar array of spots with immobilized SeqA', SeqB', and a 1:1 mixture of

SeqA':SeqB' were prepared. A 1:1 suspension of SeqA-PEG-cells labeled with PKH red and SeqB-PEG-cells labeled with PKH green was incubated on the spots for $10 \mathrm{~min}$.

After removal of unattached cells with HBSS, the surface was observed using an upright fluorescence microscope. Fig 2(d) shows SeqA-PEG-cells and SeqB-PEG-cells attached to SeqA' and SeqB'-immobilized spots, respectively, and both SeqA-PEG-cells and SeqB-PEG-cells attached to spots where a mixture of SeqA' and SeqB' was immobilized. To test whether this interaction could be inhibited, SeqA' was added to the mixture of SeqA-PEG-cells and SeqB-PEG-cells and the attachment of the cells to the substrate was examined. With the addition of SeqA', there was no attachment of SeqA-PEG-cells to the SeqA' spots, although SeqB-PEG-cells still attached to SeqB' spots (Fig. 2(e)). This inhibition assay indicated that cells were specifically attaching to the immobilized DNA via complementary DNA hybridization. The effects on cell binding to different ratios of immobilized SeqA' and SeqB' on the substrate spots were examined. Five spots of immobilized DNA were prepared using the following molar ratios of SeqA':SeqB': 4:1, 2:1, 1:1, 1:2, 1:4. A 1:1 mixture of SeqA-PEG-cells labeled with PKH red and SeqB-PEG-cells labeled with PKH green was incubated on the spots, and unattached cells were removed by washing with HBSS. 
The substrate was observed using an upright fluorescence microscope (Fig. 3(a)). The number of cells that attached depended on the ratio of the complementary DNAs that were immobilized on the spots. The ratios of SeqA-PEG-cells to SeqB-PEG-cells attached to each spot were determined from fluorescence images using ImageJ software (open circles and closed circles in Fig. 3(b), respectively). The cell ratios correlated well with the mixture ratios of SeqA' and SeqB'.

We next examined the attachment of polyDNA-PEG-cells to a pattern on the substrate; the pattern was prepared by a contact printing method using a PDMS stamp. As shown in Fig. 4(a), ledge surfaces on a PDMS stamp were coated with a solution of SeqA' or SeqB' DNA and pressed onto the gold surface. The same stamp was rotated $90^{\circ}$ and again pressed to the surface, forming a cross pattern. A 1:1 mix of SeqA-PEG-cells and SeqB-PEG-cells was applied to the immobilized DNA, incubated, and washed with HBSS. Attached cells were observed using an upright fluorescence microscope. As shown in Fig. 4(b), SeqA-PEG-cells and SeqB-PEG-cells selectively attached to the stripes containing immobilized SeqA' or SeqB' DNA, respectively, demonstrating that cells could attach via DNA hybridization to a DNA pattern prepared using a contact printing technique. 


\section{Discussion}

Cell surface modification is generally achieved three ways: by covalent conjugation to the amino groups of membrane proteins; by electrostatic interaction between cationic polymers and a negatively charged surface; and by incorporation of amphiphilic polymers into the lipid bilayer of the cell membrane by hydrophobic interactions [16]. We have studied cell surface modification using amphiphilic polymers such as PEG-lipid derivatives that incorporate spontaneously into lipid bilayers $[16,18]$.

Notably, this surface modification technique does not cause protein denaturation or have cytotoxic effects. Further, functional groups such as amino groups, maleimide, and biotin can be incorporated into the cell membrane using PEG-lipid derivatives bearing these groups [13-15].

In the present study, polyDNA was introduced into the outer cell membrane using PEG-lipid. Cell-cell attachments between either the same types of cells or different types of cells were induced by incorporating complementary DNA sequences into two cell populations (Fig. 1); when mixed, the hybridization of the complementary sequences mediated cell-cell attachment. This DNA-hybridization technique was also used to attach DNA-modified cells to immobilized DNA on a substrate (Fig. 2, 3). Antibody-antigen reactions, cell-extracellular matrix interactions, and hydrophobic 
interactions with amphiphilic polymers have all been used to immobilize cells on

surfaces [20-22]. Using these techniques, cell suspensions must be applied to each spot

to prepare arrays of cells. Not only is this a tedious and time-consuming process, cell

viability is lost during the preparation of the array. In contrast, the technique described

here is quite simple, since a suspension of cells with different DNA sequences can be

applied to surfaces that have spots of immobilized complementary DNA sequences.

Thus, this technique can be used for preparation of cell-based arrays for many types of studies.

To our knowledge, there are few previous studies that have achieved cell-cell

attachment between different kinds of cells. We previously reported the immobilization of living cells to the surface of islets of Langerhans for microencapsulation using

PEG-lipids and the biotin/streptavidin reaction [19]. It is also possible to attach feeder cells to embryoid bodies for the analysis of differentiation of ES cells into neurons

[Iwata et al., unpublished report]. The simple and versatile methods described here have many applications in both regenerative medicine and in tissue engineering. 


\section{Conclusions}

By incorporating complementary DNA sequences attached to amphiphilic PEG-lipids into the membranes of two cell populations, we induced cell-cell attachments that were mediated by DNA hybridization. This technique was also used to successfully induce cell attachment to a substrate containing immobilized DNA. This method shows promise for use in analyzing homogeneous and heterogeneous cell-cell interactions.

\section{Acknowledgements}

This study was supported in part by a Grant-in-Aid for Scientific Research (A) (No.

21240051) and a Challenging Exploratory Research grant (No. 21650118) from the Ministry of Education, Culture, Sports, Science, and Technology (MEXT) of Japan and by the Ministry of Health, Labor, and Welfare of Japan (H20-007). 


\section{References}

1. Keller G, Snodgrass HR. Human embryonic stem cells: the future is now. Nat Med 1999;5:151-152.

2. Takahashi K, Tanabe K, Ohnuki M, Narita M, Ichisaka T, Tomoda K, et al. Induction of pluripotent stem cells from adult human fibroblasts by defined factors. Cell 2007;131:861-872.

3. Takahashi K, Yamanaka S. Induction of pluripotent stem cells from mouse embryonic and adult fibroblast cultures by defined factors. Cell 2006;126:663-676.

4. Lohr M, Hoffmeyer A, Kroger J, Freund M, Hain J, Holle A, et al. Microencapsulated cell-mediated treatment of inoperable pancreatic carcinoma. Lancet 2001;357:1591-1592.

5. Wollert KC, Drexler H. Cell-based therapy for heart failure. Curr Opin Cardiol $2006 ; 21: 234-239$

6. Visted T, Bjerkvig R, Enger PO. Cell encapsulation technology as a therapeutic strategy for CNS malignancies. Neuro Oncol 2001;3:201-210.

7. Prakash S, Chang TM. Microencapsulated genetically engineered live E. coli DH5 cells administered orally to maintain normal plasma urea level in uremic rats. Nat Med 1996;2:883-887. 
8. Shoichet MS, Winn SR. Cell delivery to the central nervous system. Adv Drug

Deliv Rev 2000;42:81-102.

9. Emerich DF, Winn DR. Immunoisolation cell therapy for CNS diseases. Crit Rev Ther Drug Carrier Syst 2001;18:265-298.

10. Hao S, Su L, Guo X, Moyana T, Xiang J. A novel approach to tumor suppression using microencapsulated engineered J558/TNF-alpha cells. Exp Oncol 2005;27:56-60.

11. Lee MK, Bae YH. Cell transplantation for endocrine disorders. Adv Drug Deliv Rev 2000;42:103-120.

12. Matsumura H, Tada T. Cell fusion-mediated nuclear reprogramming of somatic cells. Reprod Biomed Online. 2008;16:51-56.

13. Miura S, Teramura Y, Iwata H. Encapsulation of islets with ultra-thin polyion complex membrane through poly(ethylene glycol)-phospholipids anchored to cell membrane. Biomaterials 2006;27:5828-5835.

14. Teramura Y, Kaneda Y, Iwata H. Islet-encapsulation in ultra-thin layer-by-layer membranes of poly(vinyl alcohol) anchored to poly(ethylene glycol)-lipids in the cell membrane. Biomaterials 2007;28:4818-4825. 
15. Teramura Y, Iwata H. Islets surface modification prevents blood-mediated inflammatory responses. Bioconjugate Chem 2008;19:1389-1395.

16. Teramura Y, Kaneda Y, Totani T, Iwata H. Behavior of synthetic polymers immobilized on cell membrane. Biomaterials 2008;29:1345-1355.

17. Totani T, Teramura Y, Iwata H, Immobilization of urokinase to islet surface by amphiphilic poly (vinyl alcohol) carrying alkyl side chains. Biomaterials 2008;29:2878-2883.

18. Teramura Y, Iwata H. Surface modification of islets with PEG-lipid for improvement of graft survival in intraportal transplantation. Transplantation 2009;88:624-630.

19. Teramura Y, Iwata H. Islet encapsulation with living cells for improvement of biocompatibility. Biomaterials 2009;30:2270-2275.

20. Kato K, Umezawa K, Miyake M, Miyake J, Nagamune T. Transfection microarray of nonadherent cells on an oleyl poly(ethylene glycol) ether-modified glass slide. Biotechniques 2004;37:444-448.

21. Ko IK, Kato K, Iwata H. A thin carboxymethyl cellulose culture substrate for the cellulase-induced harvesting of an endothelial cell sheet. J Biomater Sci Polym Ed. 2005;16:1277-1291. 
1

2

3

4

5

6

7

8

9

10

11

12

13

14

15

16

17

18

19

20

21

22

23

24

25

26

27

28

29

30

31

32

33

34

35

36

37

38

39

40

41

42

43

44

45

46

47

48

49

50

51

52

53

54

55

56

57

58

59

60

61

62

63

64

65

22. Kato K, Umezawa K, Funeriu DP, Miyake M, Miyake J, Nagamune T.

Immobilized culture of nonadherent cells on an oleyl poly(ethylene glycol)

ether-modified surface. Biotechniques 2003;35:1014-1018, 1020-1021. 
Table 1. Sequence of DNA for cell surface modification

\begin{tabular}{ll}
\hline & $5^{\prime}$ \\
polyA20 & HS-AAA AAA AAA AAA AAA AAA AA \\
polyT20 & HS-TTT TTT TTT TTT TTT TTT TT \\
SeqA & HS-TGC GGA TAA CAA TTT CAC ACA \\
SeqA' & HS-TGT GTG AAA TTG TTA TCC GCA \\
SeqB & HS-TAG TAT TCA ACA TTT CCG TGT \\
SeqB' & HS-ACA CGG AAA TGT TGA ATA CTA \\
\hline
\end{tabular}




\section{Figures captions}

Scheme 1. (a) Synthesis of DNA-conjugated PEG-DPPE (polyDNA-PEG-lipid) from maleimide-PEG-lipid and DNA-SH. (b) Schematic illustration of the interaction between polyDNA-PEG-lipid and the lipid bilayer comprising the outer cell membrane. The polyDNA-PEG-lipid inserts into the cell membrane due to hydrophobic interactions between the acyl chain and the lipid bilayer. (c) Schematic illustration of cell-cell attachment through DNA hybridization between complementary polyDNA-PEG-lipids incorporated into the outer cell membranes.

Figure 1. Cell-cell attachment via DNA hybridization between complementary polyDNA-PEG-lipids on cell surfaces. CCRF-CEM cells incorporated polyA20-PEG-lipid into the outer cell membranes. Cells were observed by a confocal laser scanning microscope after polyA20-PEG-lipid modified CCRF-CEM cells were further treated with (a): FITC-labeled polyT20 and (b): FITC-labeled polyA20. (c): Cell-cell attachment between polyA20-PEG-lipid modified CCRF-CEM cells labeled with PKH red and polyT20-PEG-lipid modified CCRF-CEM cells labeled with PKH green in culture medium (cells were mixed in a 1:1 ratio). Cells were observed over time using a confocal laser scanning microscope and a phase contrast microscope. (d): 
Cell-cell attachment between polyA20-PEG-lipid modified CCRF-CEM cells and polyT20-PEG-lipid modified GFP-HEK293 cells (cells were mixed in a 1:1 ratio). (e):

Control experiments for cell-cell attachment by surface modification with polyDNA-PEG-lipid. (e-1): A mixture of CCRF-CEM cells labeled with PKH red and CCRF-CEM cells labeled with PKH green (no polyDNA-PEG-lipid modification). (e-2): PolyT20-PEG-lipid modified cells. (e-3): A mixture of CCRF-CEM cells labeled with PKH green and GFP-HEK293 cells after rotation culture at $100 \mathrm{rpm}$ (no polyDNA-PEG-lipid modification).

Figure 2. Immobilization of polyDNA-PEG-lipid modified cells to a complementary polyDNA' modified surface. (a): Scheme for preparation of DNA'-patterned substrate and immobilization of polyDNA-PEG-lipid modified cells. (b): Immobilization of polyT20-PEG-lipid modified CCRF-CEM cells labeled with PKH green to a single spot with immobilized polyA20-SH. The spot on the substrate surface was observed using an upright fluorescence microscope. (c): Attachment of polyT20-PEG-lipid modified CCRF-CEM cells to spots with immobilized polyA20-SH (solid lines) and polyT20-SH (dotted lines). The spots were observed using a stereomicroscope. (d): A mixture of SeqA-PEG-lipid modified CCRF-CEM cells and SeqB-PEG-lipid modified 
CCRF-CEM cells was incubated on DNA-immobilized spots where SeqA' (top right), SeqB' (bottom left), or a 1:1 mixture of SeqA' and SeqB' (top left and bottom right) were immobilized. (e): Inhibition assay for (d). A solution of SeqA'-SH was added to the mixture of SeqA-PEG-cells and SeqB-PEG-cells in advance and then the cells were incubated on the spots.

Figure 3. Varying the ratios of immobilized SeqA' and SeqB' DNA in spots on the substrate surface and the effect on cell attachment. A 1:1 mixture of SeqA-PEG-cells labeled with PKH red and SeqB-PEG-cells labeled with PKH green were applied to the spots. (a): The surface was observed using an upright fluorescence microscope. (b): The ratios of SeqA-PEG-cells (open circles) and SeqB-PEG-cells (closed circles) attached to each spot were determined from fluorescence images using ImageJ software. The composition of cells are plotted against the SeqA':SeqB' ratios in the spots.

Figure 4. Immobilization of cells on a patterned substrate prepared by a contact printing method using a PDMS stamp. (a): SeqA'-SH and SeqB'-SH were immobilized (red and green lines, respectively) in the pattern shown here. (b): A 1:1 mixture of SeqA-PEG-lipid cells labeled with PKH red and SeqB-PEG-lipid cells labeled with 
PKH green was applied to the patterned substrate containing immobilized DNA. The substrate was observed using an upright fluorescence microscope. 


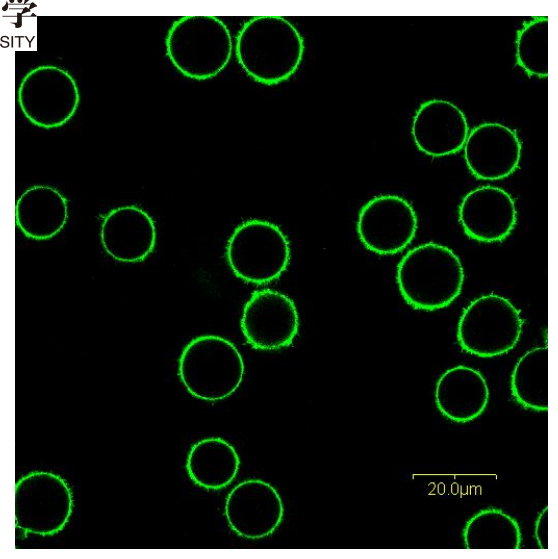

(b)

KERENAN II

(c)

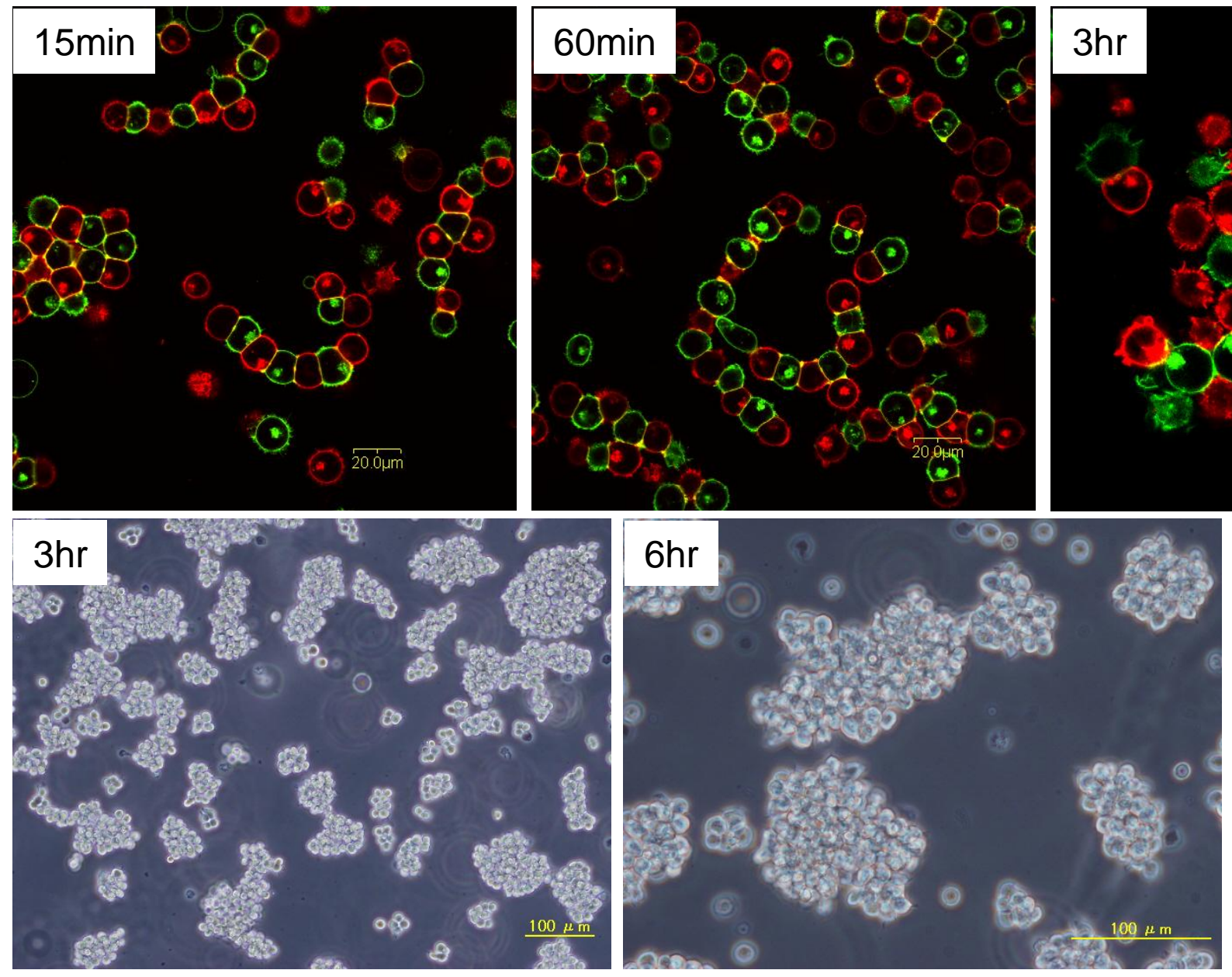

Figure 1 
$\mathbf{F}=$ 京都大学

kroro yivive

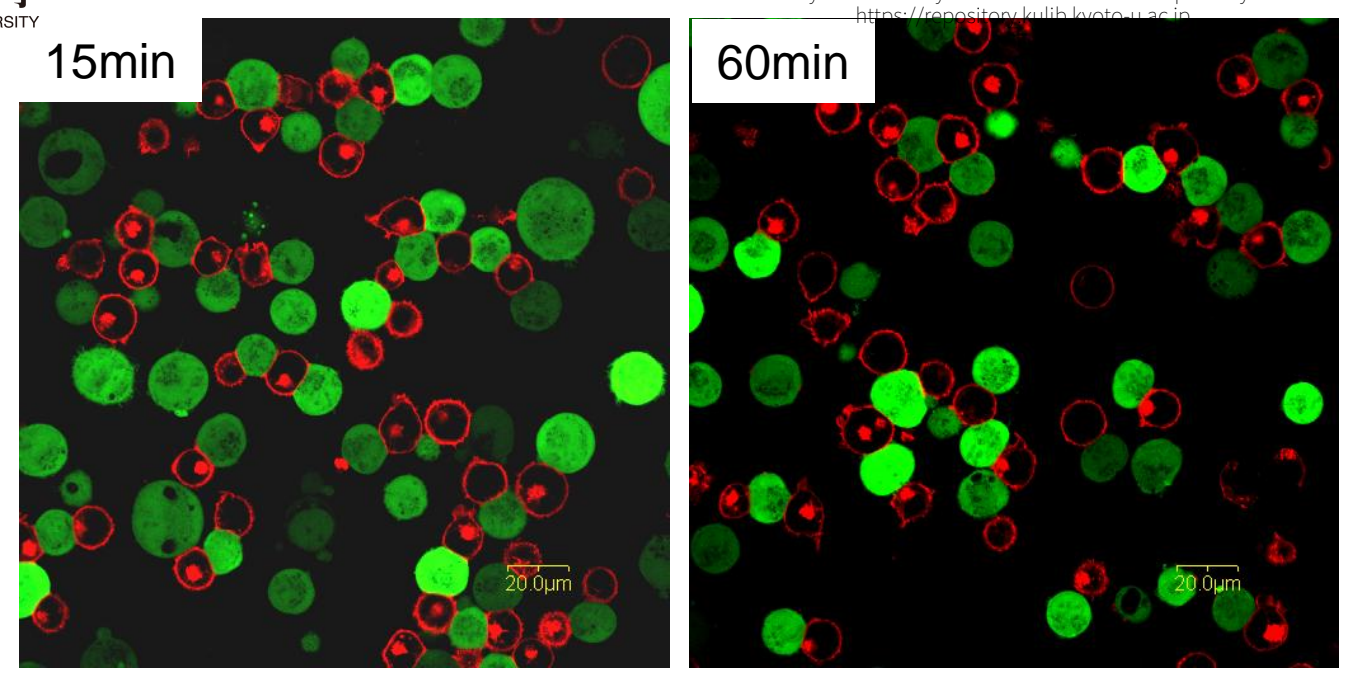

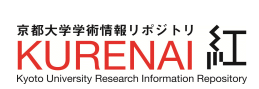

(e)

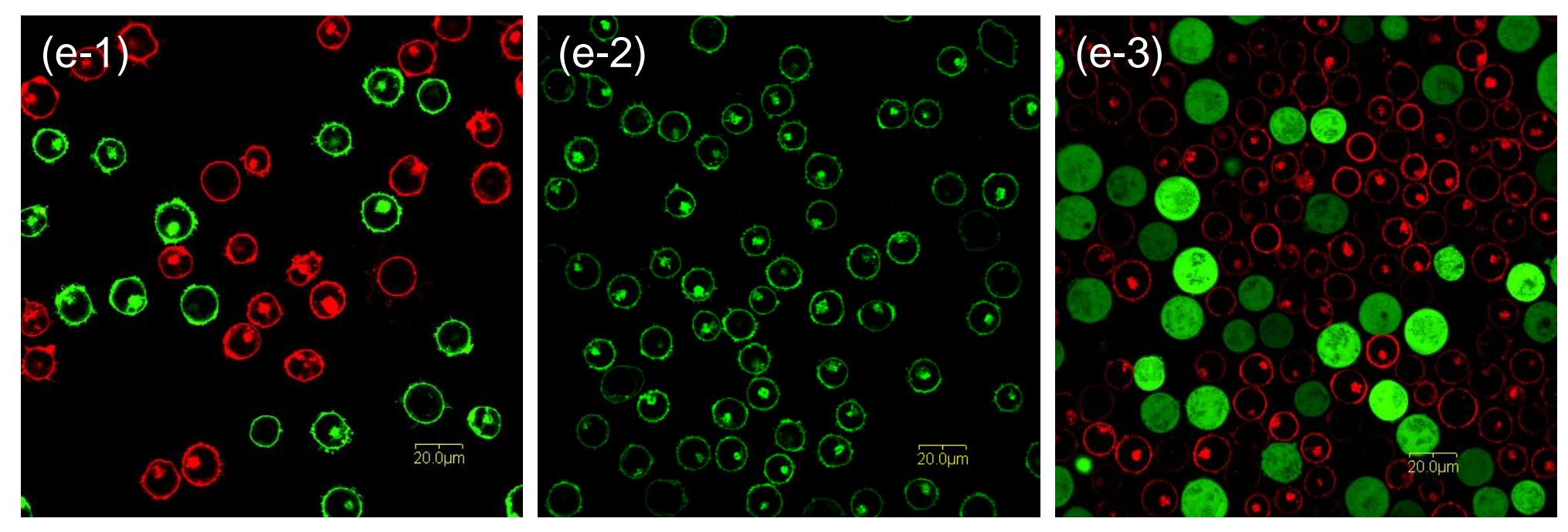

Figure 1 


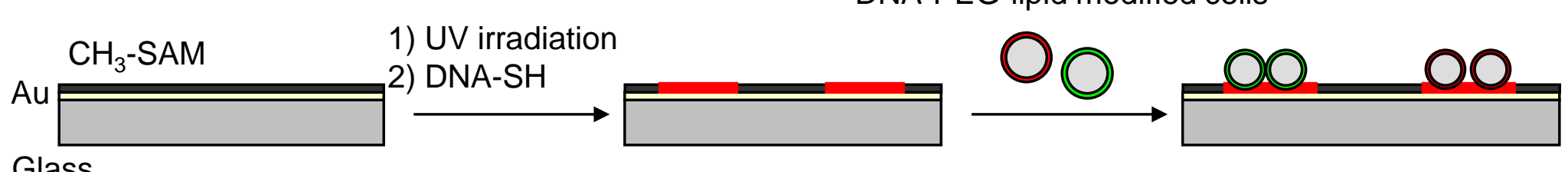

(b)

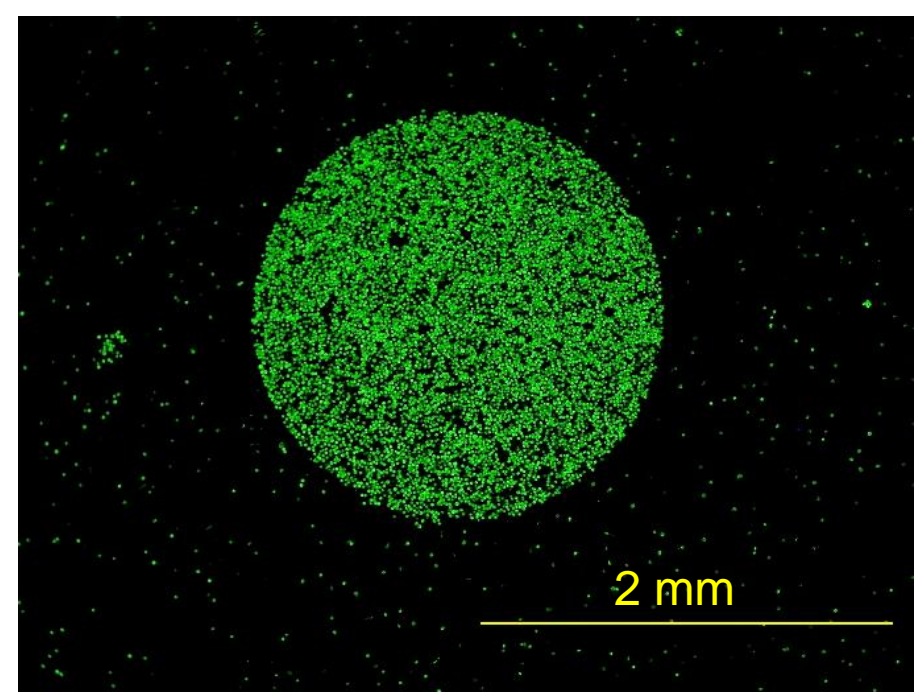

(d)

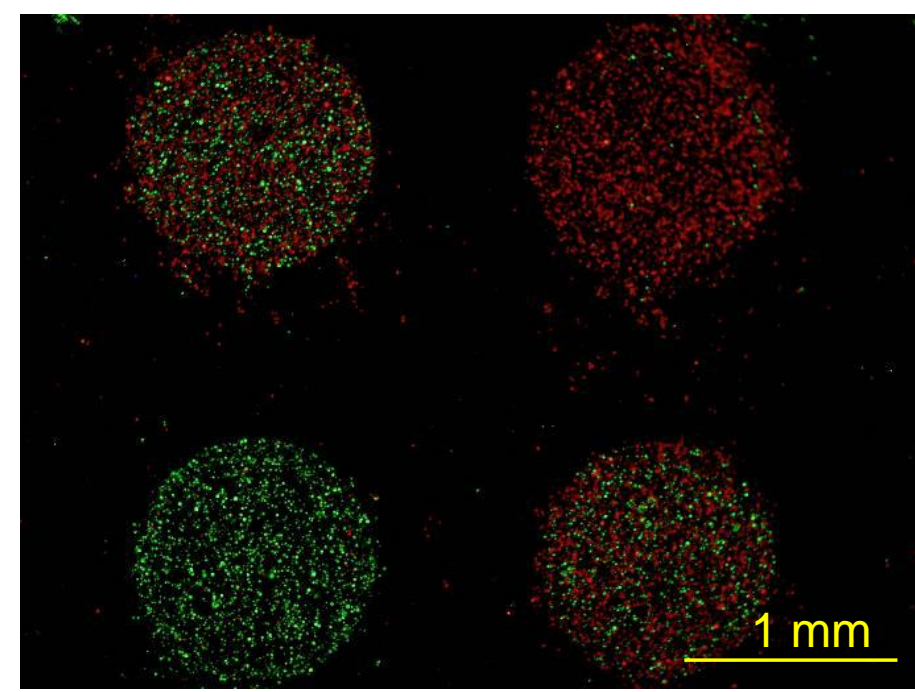

(c)
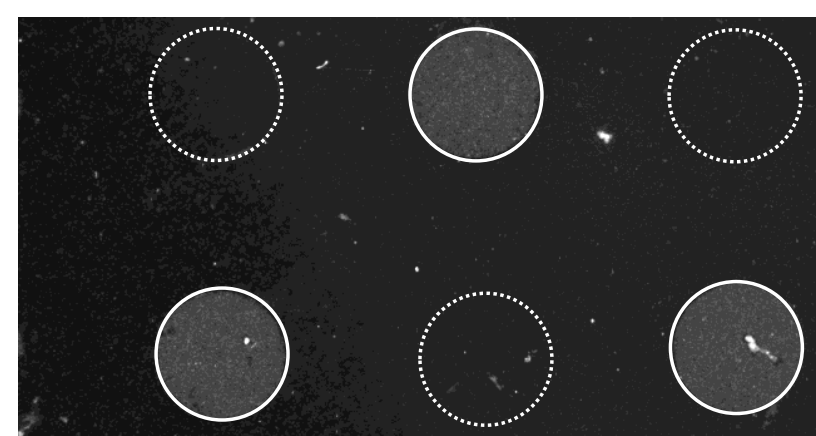

(e)
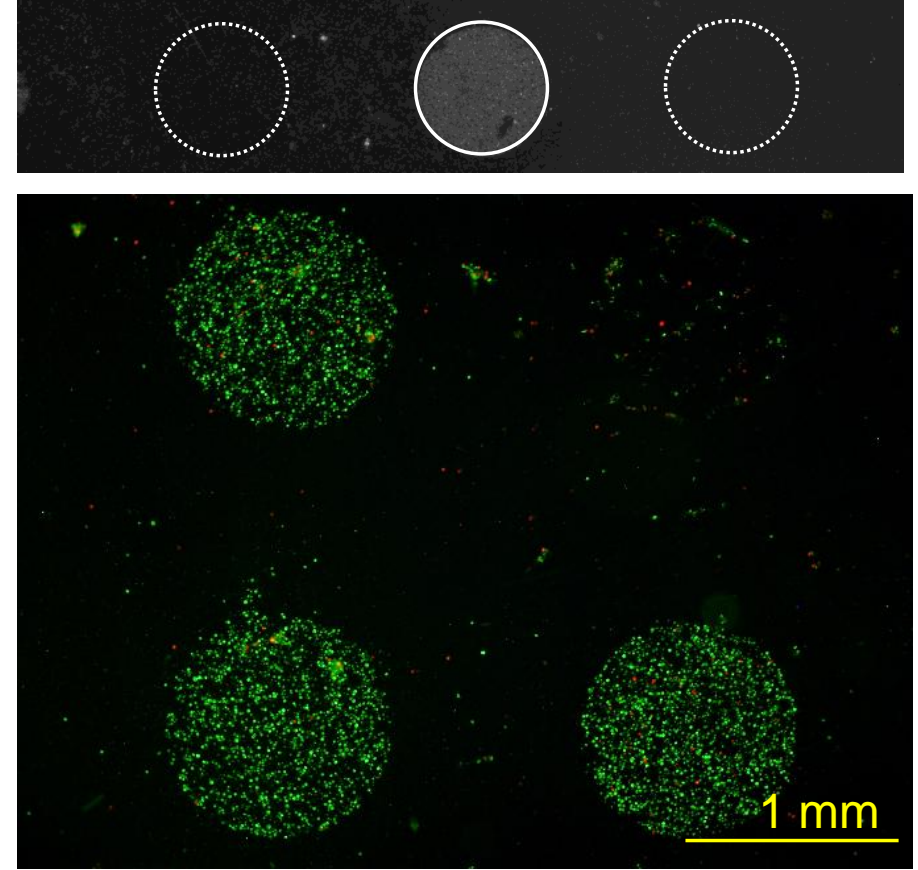

Figure 2 


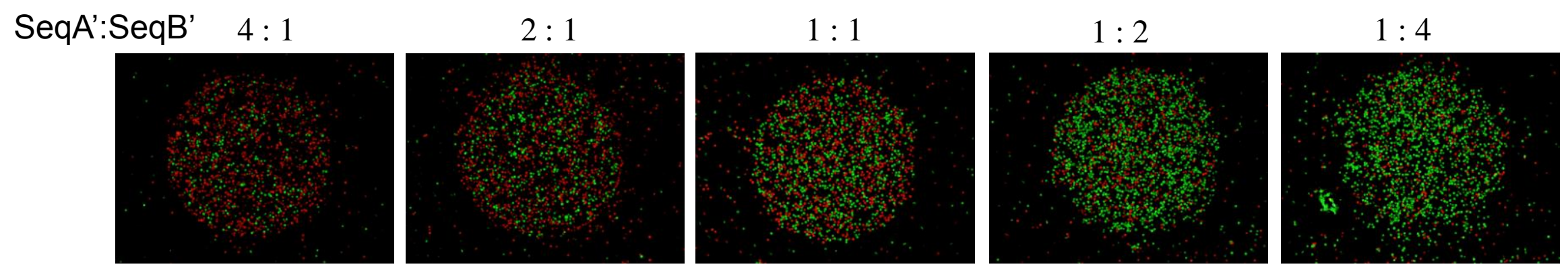

(b)

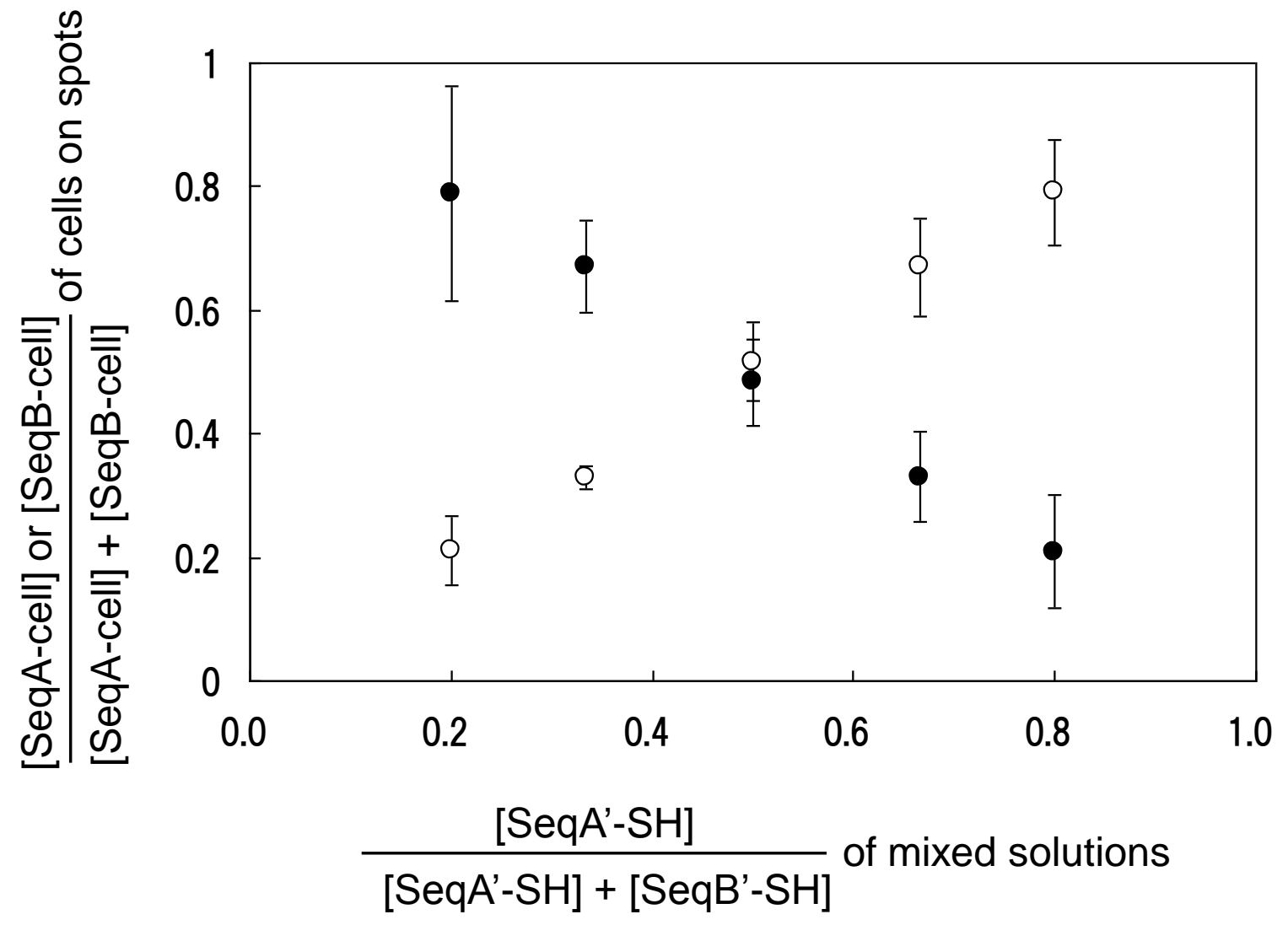

Figure 3 
(a)

(b)
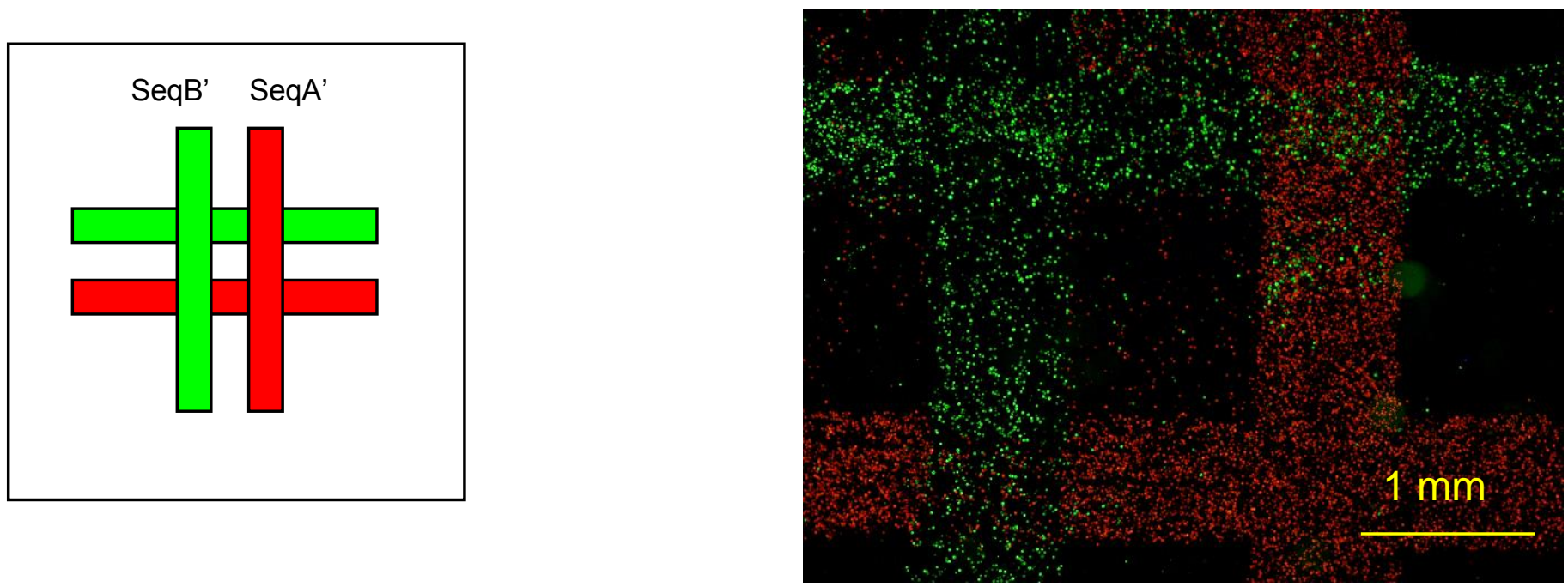

Figure 4 


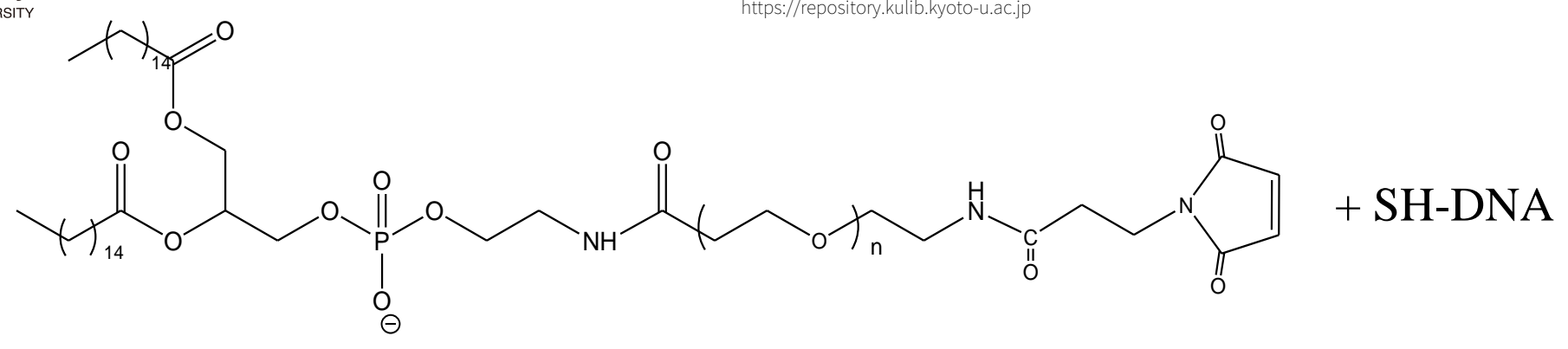

(b)

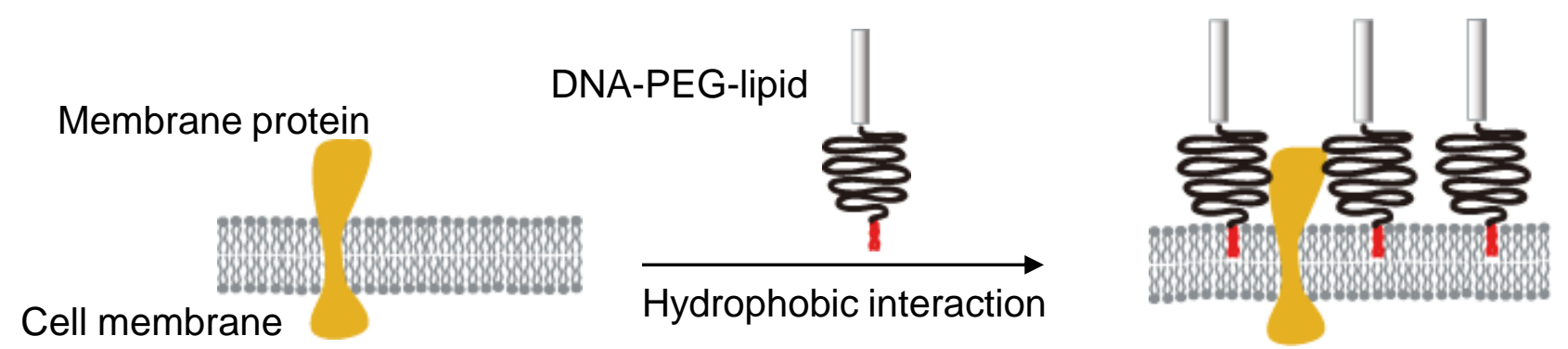

(c)

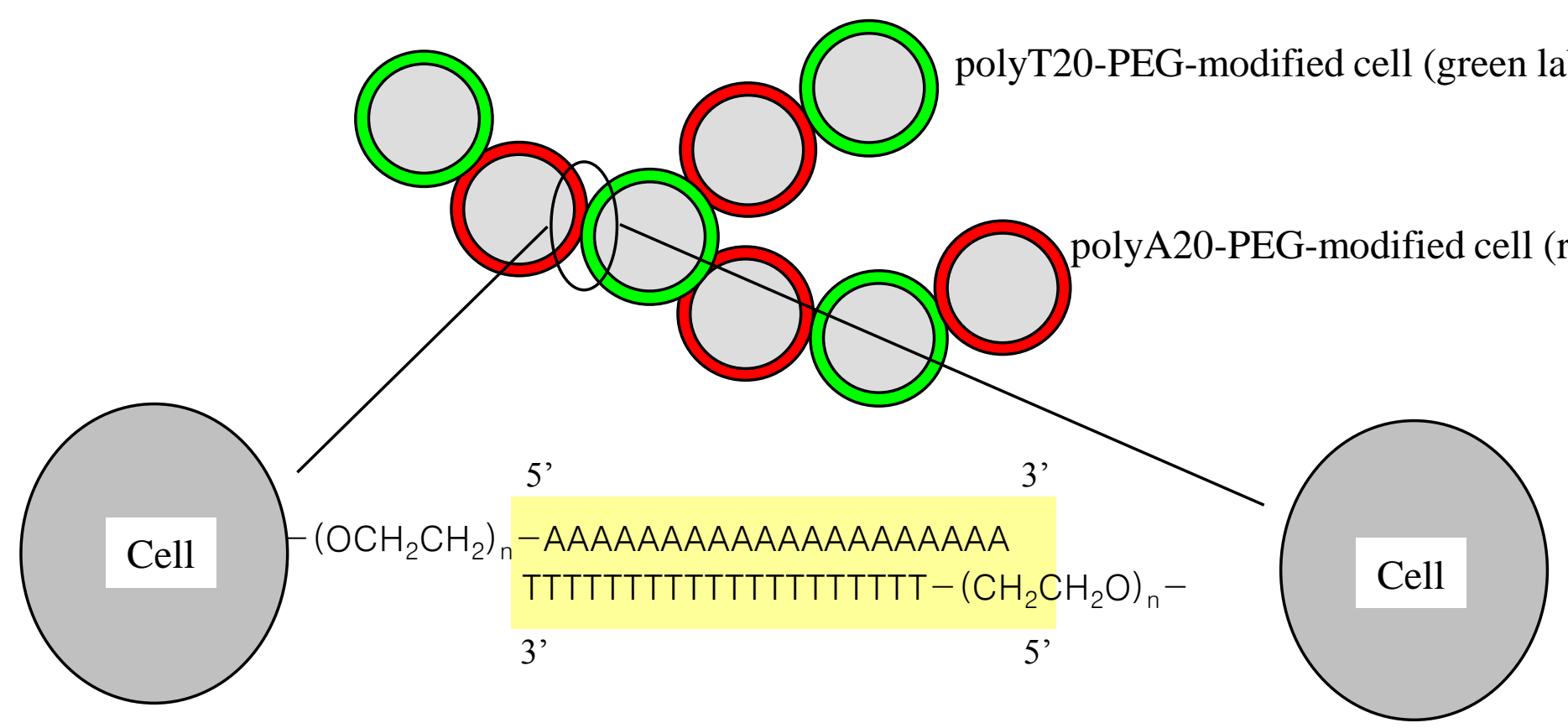




\title{
Supplementary information
}

\section{Control of cell attachment through polyDNA hybridization}

Yuji Teramura $^{\mathrm{a}}$, Hao Chen ${ }^{\mathrm{b}}$, Takuo Kawamoto ${ }^{\mathrm{a}}$ and Hiroo Iwata ${ }^{\mathrm{b} *}$

\author{
${ }^{a}$ Radioisotope Research Center, Kyoto University, Yoshida-Konoe-Cho, Sakyo-ku, \\ Kyoto, 606-8501, Japan, ${ }^{\mathrm{b}}$ Department of Reparative Materials, Institute for Frontier \\ Medical Sciences, Kyoto University, 53 Kawara-Cho, Shogoin, Sakyo-ku, Kyoto, \\ 606-8507, Japan. \\ * Address correspondence and reprint requests to Hiroo Iwata, Ph.D. \\ E-mail: iwata@frontier.kyoto-u.ac.jp, PHONE/FAX: +81-75-751-4119
}

We examined cell-cell attachment when the ratio of

polyA20-PEG-cells:polyT20-PEG-cells was 2:1, 4:1, and 10:1. Supplementary Fig. 1

shows images of the resulting cell attachment (observed using a fluorescence

microscope). No large aggregates of cells were observed when the mixture ratio was

$1: 1$, although we observed small clusters in which 1-3 polyA20-PEG-cells attached to a

polyT20-PEG-cell. It seemed that polyA20-PEG-cells and polyT20-PEG-cells could not

make contact. Intercellular attachment did not occur between the same kinds of cells.

When the ratio of polyA20-PEG-cells to polyT20-PEG-cells was 2:1, 4:1, and 10:1,

there were about 3 polyA20-PEG-cells attached to each polyT20-PEG-cell, indicating

that changing the cell ratio did not change the attachment ratio. The equivalent cell 
number is important for the cell-cell attachment reaction. The ratio of the number of attachements between polyA20-PEG-cells and polyT20-PEG-cells per total number of attachments in all attaching cells was 1, indicating attachment between polyA20-PEG-cells and polyT20-PEG-cells. Thus, it is possible to induce cell-cell attachment by the surface modification with PEG-lipid and control cell-cell attachments by varying the mixture ratio.

We also studied the effects of mixed cell ratios on attachment to a substrate upon which spots were prepared using a 1:1 mixture of SeqA' and SeqB'. Mixtures of SeqA-PEG-cells to SeqB-PEG-cells at ratios of 4:1, 2:1, 1:1, 1:2, and 1:4 were applied to the spots, and the spots were observed by an upright fluorescence microscope (Supplementary Fig. 2 (a)). The ratio of SeqA-PEG-cells to SeqB-PEG-cells attached to each spot were determined from fluorescence images using ImageJ software (Supplementary Fig. 2 (b)). The ratios of SeqA-PEG-cells to SeqB-PEG-cells on spots were determined from fluorescence images using ImageJ software. The cell ratios correlated well with the ratios of SeqA-PEG-cells and SeqB-PEG-cells that were applied to the spots. 
(a)
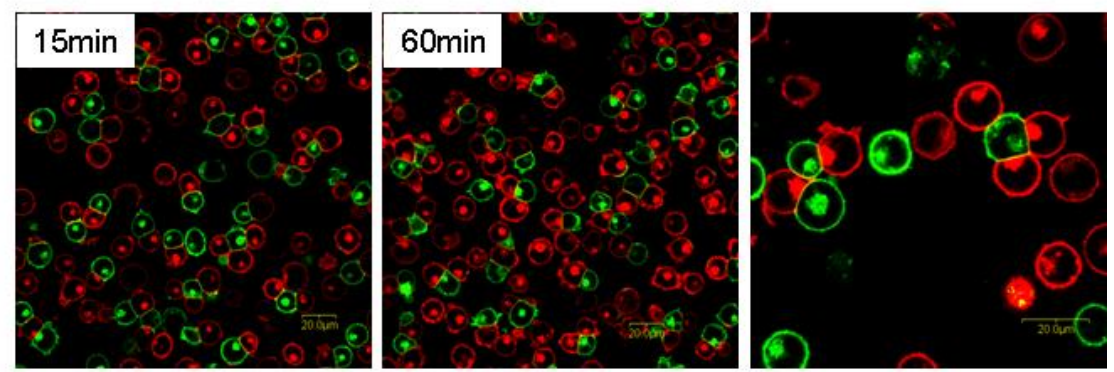

(b)
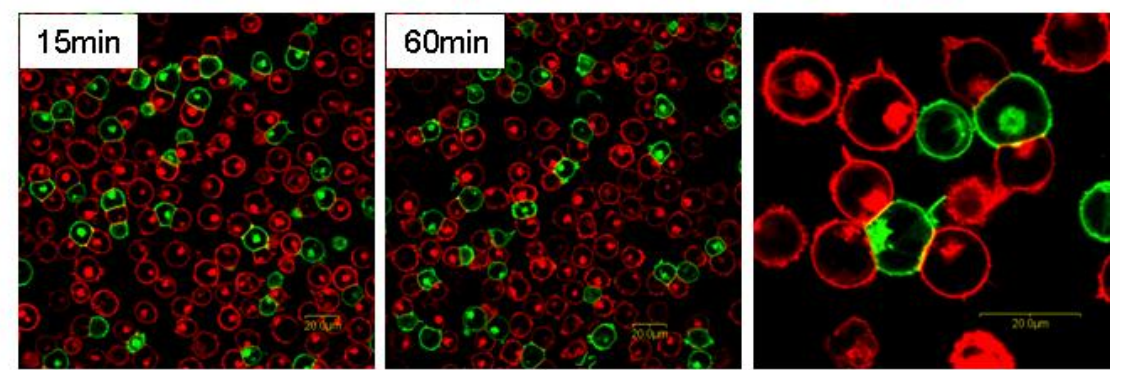

(c)
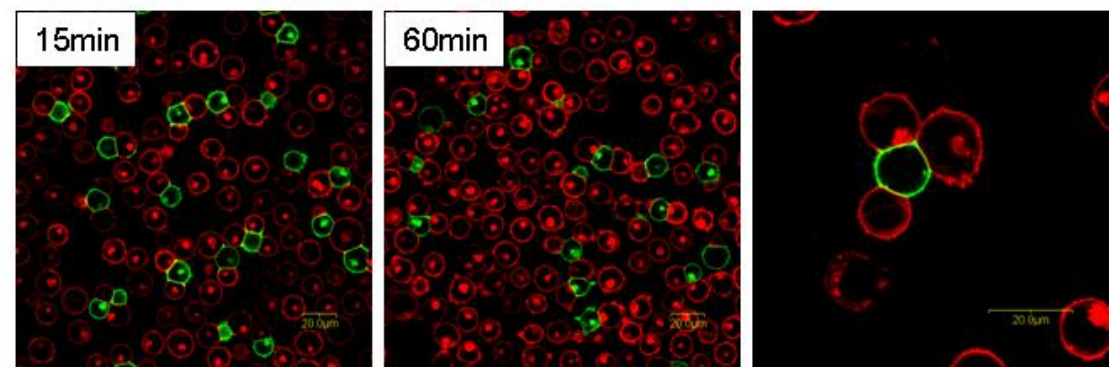

Supplementary Figure 1. Effect of the ratio of polyA20-PEG-cells (labeled with PKH

red) to polyT20-PEG-cells (labeled with PKH green) on cell-cell attachment.

PolyA20-PEG-cells and polyT20-PEG-cells were mixed at the following ratios and attachment was observed over time: (a) 2:1, (b) 4:1, and (c) 10:1. The images were acquired using a confocal laser scanning microscope. 
(a)
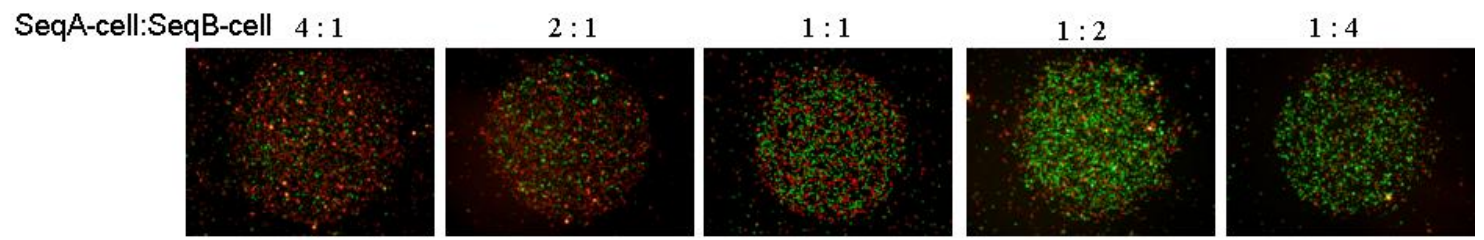

(b)

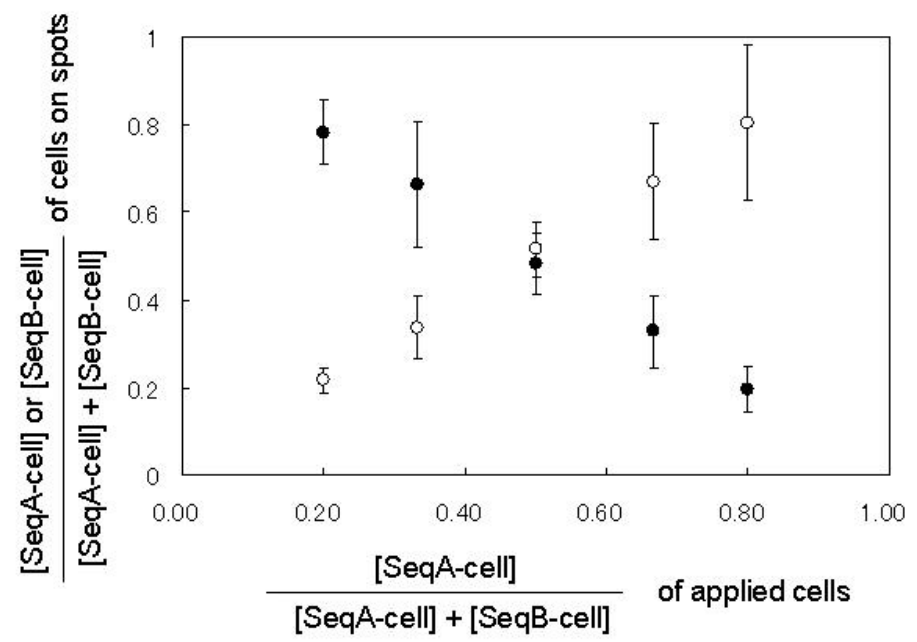

Supplementary Figure 2. Effect of varying the ratio of SeqA-PEG-cells and

SeqB-PEG-cells in the mix of cells applied to immobilized DNA in the attachment assay. A 1:1 mixture of SeqA'-SH and SeqB'-SH was immobilized to spots on the substrate. Cell suspensions of SeqA-PEG-cells labeled with PKH red and SeqB-PEG-cells labeled with PKH green were applied to the spots; the suspension had cells mixed in the following ratios: 4:1, 2:1, 1:1, 1:2, and 1:4. (a): Images acquired using an upright fluorescence microscope. (b): The ratio of SeqA-PEG-lipid cells (open circles) to SeqB-PEG-lipid cells (closed circles) on each spot were determined 
from fluorescence images using ImageJ software. The composition of cells attached to each spot are plotted against the ratio of SeqA-PEG-cells in the applied cell mixture. 